\title{
Novel Double Lumen Catheter for Drug Delivery at the Skin- Catheter Interface
}

\section{Antonio Peramo}

Department of Oral and Maxillofacial Surgery, University of Michigan, Ann Arbor, Michigan 48109

\begin{abstract}
This paper shows the technical feasibility of using a modified double lumen catheter for the delivery of fluids at the skin-catheter interface in an in vivo rabbit model. The modified catheter permits the extrusion of suspensions at the site of contact between the skin and the modified catheter (exit site). The modification consisted in the addition of a second lumen, exterior to the original one of the catheter, and of shorter length, ending in an extrusion port placed sub dermally. An external fluid pump was attached to the catheter and the whole system was tested in vitro in human skin, in a cadaver model and in an in vivo rabbit model. Experiments were performed by delivering solutions of methylene blue in a controlled manner to the subcutaneous area to analyze the skin response to the process. Results indicate that this technique of pumping the material is effective in producing observable epithelial changes in the short term. The modification of the catheter addresses catheter exit site lack of integration of skin and works as a model that can be implemented in a number of percutaneous devices for long-term implantation.
\end{abstract}

Keywords: Percutaneous; Skin biomaterial; Double lumen; Bio integration

\section{Introduction}

The current trend in using percutaneous devices in contemporary medicine and the increased dependence on advanced treatments with technologically improved delivery systems will produce a sustained growth in the number of patients undergoing procedures where a skin-implant interface will be present $[1-3,12]$. Increasingly, these bio interfaces will also become long-term or permanent interfaces. Typical complications originating from the use of these devices are break-down of the skin and serious infections which makes more difficult the formation of a long-term, stable dermal and epidermal sealing around the implants. These devices show high variability in their intrinsic properties, with a disparate number of material composition, surface structure, porosities and topologies $[4,13]$. Successfully addressing the problems originating at this bio interface will also be helpful to avoid or reduce complications in surgical procedures that require long-term permanent access to internal areas after total implantation $[5,6,15]$.

To start addressing these issues with per cutaneous devices, we have previously presented two in vitro model systems to provide proof-of-concept of the feasibility of delivering materials at the skin-percutaneous device interface [7-9], permitting the analysis of the interface between skin and external devices. They were used to observe changes of the skin in contact with implants in the presence or absence of a variety of materials in solution, which were delivered manually and discontinuously or on a continuous basis using a fluid pump. We showed that the delivery process induced biological changes at the interface. Standard methods to address the problem typically involve coating the surfaces of the percutaneous devices with antimicrobial or other products. Those systems provide a nondynamic, non-repeatable method of delivering the products to the interface. Our hypothesis is that the delivery of specific biomaterials at this interface will create a dynamic, slowly flowing matrix for skin bio integration and the local administration of drugs or antimicrobials. Our essential concept is to incorporate a reservoir of material that can be slowly extruded at the implant-skin interface to protect the underlying tissue layers from the possibility of infection and inflammation or introduce regenerative materials or therapies, as discussed previously [10].
To further advance in solutions to help bioengineer the skinimplant interface, here we present a simplified examination of the implementation of a technique using a novel double lumen catheter to deliver solutions in a controlled manner to the interface. In vitro human skin, a rabbit cadaver model and an in vivo rabbit model were used. The modifications were engineered to permit the dynamic delivery of liquids to the skin catheter area. Our expectation is that the modification of the catheter by the addition of a secondary, but shorter lumen will be helpful in controlling clinical conditions that may arise in the implant area over time, including control of inflammation or reduction of infections. In these preliminary experiments the catheters are inserted sub dermally, protruding from the skin and left in place for short periods of time while methylene blue solutions are pumped. In these preliminary experiments the objective is to determine the approximate areas affected by the pumping process and whether this system can be implemented in vivo.

Similar technologies are not available in current clinical practice. Some commercial catheters contain a single lumen while others have a double or triple lumen, but their purpose is to deliver solutions to the venous system, the peritoneum or other internal body areas, not to the areas of contact between the catheter and the skin or subcutaneous tissue. At this stage of the development, this system does not address luminal infections, which are normally addressed by coating the interior surfaces of the catheter with antimicrobials [16]. Some commercially available catheters have specially designed cuffs, containing antimicrobial compounds. The main drawback of both solutions is that they provide a non-dynamic, non-repeatable method of delivering the antimicrobials to the affected areas and that only one type of product, in this case, antimicrobials, can be delivered at the interface.

Corresponding author: Antonio Peramo, Department of Oral and Maxillofacial Surgery, University of Michigan, Ann Arbor, Michigan 48109, Email: aperamo@ umich.edu

Accepted October 23, 2010; Published November 08, 2010

Citation: Peramo A (2010) Novel Double Lumen Catheter for Drug Delivery at the Skin-Catheter Interface. J Tissue Sci Eng 1:102. doi:10.4172/2157-7552.1000102

Copyright: (C) 2010 Peramo A. This is an open-access article distributed under the terms of the Creative Commons Attribution License, which permits unrestricted use, distribution, and reproduction in any medium, provided the original author and source are credited. 
The ultimate goal is then the development of a system that will allow the physician to use an externally connected device for the delivery of solutions of his/her choice. This system will permit the treatment of conditions that the patient may develop over time around the interface without the need to open the area around the implant and reduce the risk of implant failure and retrieval. The results with the rabbit model presented here indicate that this modified device may be useful in humans for control, maintenance and repair of the skin-implant interface.

\section{Materials and Methods}

\section{Experimental design}

Figure 1 shows the modified double lumen catheter system. It consisted of an external pumping system composed of reservoir and pump, tubing and an external port and a subcutaneous delivery port. Two types of tubing were used. The outer tube (Silicon Rubber $4 \mathrm{~mm}$ OD, McMaster-Carr) connected the pump to the external port
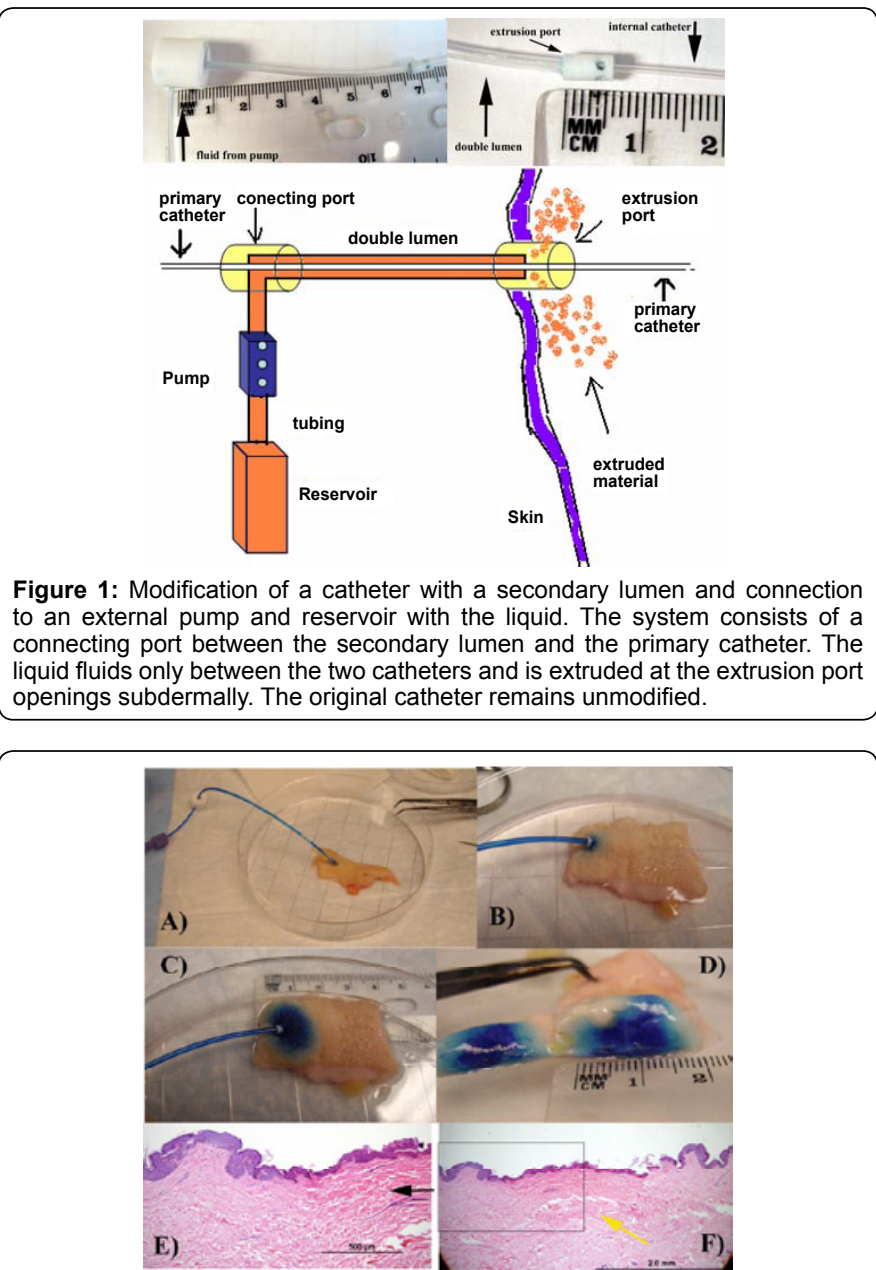

Figure 2: These images (A, B, D and D) show the process of delivering $10 \%$ methylene blue to human skin using the double catheter with the extrusion port for 20 hours at an average rate of $0.6 \mathrm{ul} / \mathrm{min}$. The liquid flows in the lumen between the internal and external tubes and is released at the exit site. The original functionality of the internal catheter (i.e. peritoneal catheter or other) is maintained and not affected by this process. In the second row, histological analysis showed focal disruption in the epidermis accompanied by minimal focal epidermal necrosis as seen on image $\mathbf{E}$. Visible on $\mathbf{F}$ is the overall area affected by the dye, reaching a depth of about $1 \mathrm{~mm}$ and indicated by an arrow. Overall, the changes in this sample were minimal to mild and there was no significant epidermal or dermal damage.

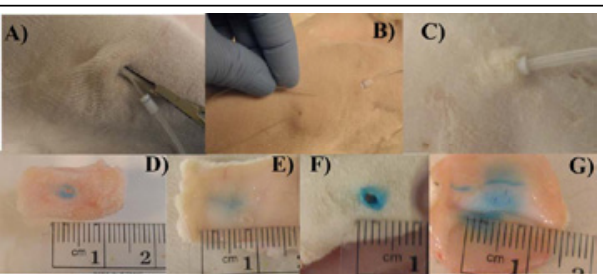

Figure 3: The image below shows the procedure to insert the catheter on the dorsum of a rabbit (A, B and $\mathbf{C}$ ) and distribution and testing time (D, E, F and G). To simplify the process, in this procedure the internal catheter is placed subdermally. To avoid additional injury to the exit site the catheter is placed without sutures. In second row, testing times and concentrations indicated that $1 \%$ methylene blue diffused into the epidermis, dermis and reached the panniculus muscle of the rabbit in the cadaver model. Over a 2 hour pumping period at $0.1 \mathrm{ul} / \mathrm{min}(\mathbf{D}$ and $\mathbf{E}$ ), the distribution of the dye formed a $1 \mathrm{~mm}$ corona around the exit site. At $1 \mathrm{ul} / \mathrm{min}(\mathbf{F}$ and $\mathbf{G}$ ) the corona extended $2 \mathrm{~mm}$ with more methylene blue reaching the dermis. While the distribution around the epidermis always appears to be circular in shape, in the dermis the diffusion depends on the specific structural features of the tissue, and then the dye is present in irregular shapes. This distribution will obviously be different for other compounds.

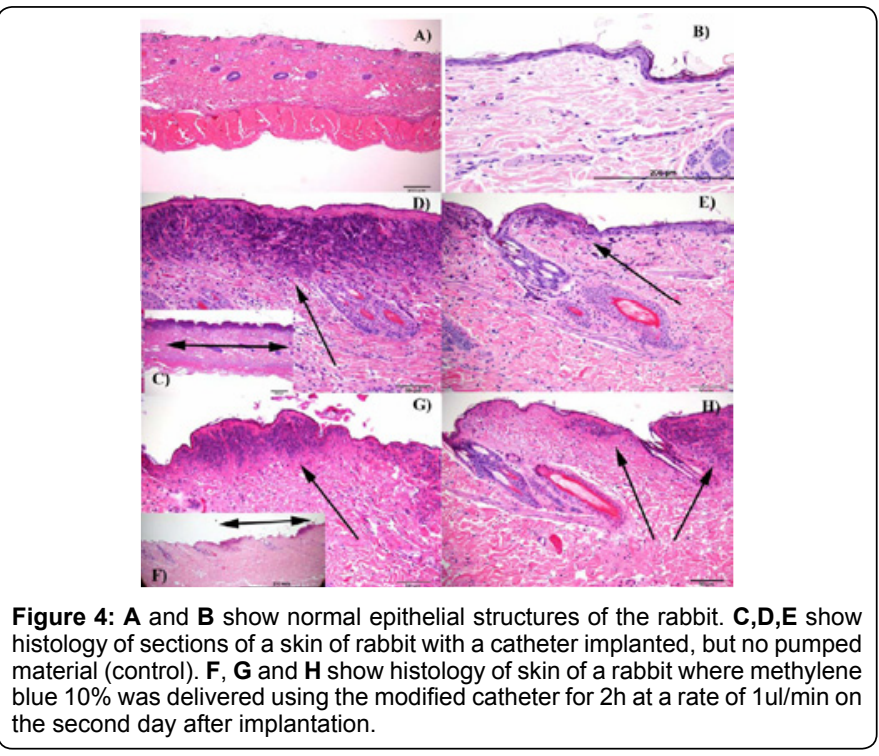

and continued to the delivery port. The internal tubing connected the external port to the delivery port. The liquid suspensions flew using the open space between the two tubes, which was a cylindrical section of 200 micrometer radius. The inner catheter was left empty. The delivery port was a cylinder made of commercial Teflon, radius $1 \mathrm{~cm}$ and length $2 \mathrm{~cm}$ with three side openings for the release of liquids.

Preliminary experiments were performed with human skin in vitro obtained from discarded surgeries (Figure 2) followed by animal experiments in a cadaver model and in vivo (Figures 3 and 4). To adjust the system around the animals and prevent the rabbits from scratching catheters exit sites, rabbits wore a jacket (Lomir Biomedical, Quebec, Canada) with a pocket, where the device was placed. In the cadaver model a $1 \%$ methylene blue (MW 320) solution in distilled water was used, while in the human skin specimens and in vivo rabbit a $10 \%$ methylene blue solution was used. In the results presented, the pump used a pulsatile system connected to a timer (Crouzet USA, Texas) that discharged specified volumes of liquid during on periods and stopped during off periods of time. For the human skin experiments this produced an average flow of $0.6 \mu \mathrm{l} / \mathrm{min}$. For the rabbit cadaver model the average fluid rates were $0.1 \mu \mathrm{l} / \mathrm{min}$ and $1 \mu \mathrm{l} / \mathrm{min}$ and in 
the in vivo rabbit model the average pumping rate was $0.4 \mu \mathrm{l} / \mathrm{min}$. The tubing and pump were primed with the suspensions before the start of the experiment. Experiments performed in cadaver models of the animals provided basic information to determine the basic surgical procedures described and to obtain control skin sections and approximate distribution of the suspensions.

\section{Animal surgery}

The cadaver model and in vivo experiments were performed with New Zealand white rabbits weighing between 1.8 and $2.4 \mathrm{~kg}$ in accordance with the University of Michigan animal research facility guidelines. Animals were acclimated to the animal housing facility for at least 48 hours. Rabbits were fed a standard diet and were given water ad libitum. Rabbits fasted for 2 hours and were anesthetized by an intramuscular injection of dexdormitor $(0.15 \mathrm{mg} / \mathrm{kg})$ and maintained with isoflurane. Dorsum fur was removed with clippers and disinfected with chlorhexidine. A small incision was made to open the skin enough for the insertion of the modified catheter, as shown in Figure 3A. The procedure consisted in pulling the small diameter catheter sub dermally and securing the end to the skin approximately $7 \mathrm{~cm}$ from the exit site (to conform to current terminology used in the literature, we use the term "exit site" to describe the skin area where the delivery port is located). The terminal part of the internal catheter was secured with a knot without the need of suture. The delivery port was then placed sub dermally, as shown in Figure 3C, without suture. Two catheters were placed on the animals: one connected to the pumping system, the other used as control with no pump attached (catheter only). No antibiotics or products were given to the animal and the tissues around the area of the exit site were left to readjust mechanically to the delivery port for 24 hours before releasing any material in the in vivo model. A similar configuration was implemented in the cadaver rabbit model. In this later case, the catheter was placed immediately after death and pumping was performed for a maximum of two hours.

\section{Analysis of soft tissue specimens}

At the end of the pumping period specimens were analyzed for extension and distribution of the fluids. Basic pathology analysis was also done. For histological analysis, tissues were fixed in $10 \%$ phosphate buffered formalin for $24 \mathrm{~h}$, routinely dehydrated and paraffin embedded. Serial sections were obtained at $5 \mu \mathrm{m}$. Histological evaluation of specimens was performed with haematoxylin and eosin (H\&E).

\section{Results}

The configuration of the catheter system and the volumes perfused allowed the liquids to reach the fascia and muscle areas. No adhesive was used to bind the catheters to the subcutaneous tissues and no anti-microbials were used and the exit sites were free of bacterial infection. During the experiments, visual morphological changes of the tissues around the catheter were controlled. No gross morphological changes were observed during the experiments among the control and experimental specimens. The only noticeable change was the diffusion of methylene blue in all skin specimens. Animals were controlled to prevent scratching or substantial mechanical disturbances around the catheter exit site. This was done to prevent the occurrence of inflammatory processes not related to the presence of the catheter or the pumping process itself. Figure 2 shows an example of human skin with the catheter delivering $10 \%$ methylene blue for 20 hours at a rate of $0.6 \mu \mathrm{l} / \mathrm{min}$, for a total volume of $720 \mu l$. The liquid flows in the lumen between the internal and external tubes and is released at the exit site. The original functionality of the internal catheter (i.e. peritoneal catheter or other) is maintained and not affected by this process. The extrusion port was left semi-inserted to simulate the process where skin has to attach to external ports, as is common in peritoneal dialysis. In these static conditions the dye distributed radially from the port in the epidermis and diffused down to the dermis. There was a gradient of diffusion where the dye concentrated (intense blue) and dye was less intense far from the exit site (light blue). These areas extended up to $1 \mathrm{~cm}$ from the entrance site in the configuration tested. Histological analysis of the human skin showed focal disruption in the epidermis accompanied by minimal focal epidermal necrosis as seen on Figure 2E. Localized interstitial cell psychosis could also be observed. Visible on Figure $2 \mathrm{~F}$ is the overall area affected by the dye, reaching a depth of about $1 \mathrm{~mm}$ and indicated by an arrow. Overall, the changes in this sample were minimal to mild and there was no substantial epidermal or dermal damage.

As seen in Figure 3, testing times and concentrations indicated that $1 \%$ methylene blue diffused into the epidermis and dermis and reached the panniculus muscle of the rabbit in the cadaver model. Over a 2 hour pumping period at $0.1 \mu \mathrm{l} / \mathrm{min}$ (Figure 3D and 3E, total volume $12 \mu \mathrm{l}$ ), the distribution of the dye formed a $1 \mathrm{~mm}$ corona around the exit site. At $1 \mu \mathrm{l} / \mathrm{min}$ (Figure $3 \mathrm{~F}$ and $3 \mathrm{G}$ total volume $120 \mu \mathrm{l}$ ) the corona extended $2 \mathrm{~mm}$ with more methylene blue reaching the dermis. While the distribution around the epidermis always appears to be circular in shape, in the dermis the diffusion depends on the specific structural features of the tissue, and then the dye is present in irregular shapes. It is expected this distribution will be different for other compounds.

Figure 4 is a composite figure with pictures of the histology of the specimens taken from the in vivo rabbit model. Figure $4 \mathrm{~A}$ and Figure $4 \mathrm{~B}$ show normal epithelial structures of the rabbit skin which has a very thin epidermis ( 1 or 2 cells). Figures 4C, 4D and 4E show sections of control catheter implanted but no pumped material (catheter control). Figures 4F, 4G and 4H show histology of sections where methylene blue $10 \%$ was delivered using the modified catheter at an average rate of $0.41 \mu / \mathrm{min}$ for 4 hours (total volume $96 \mu \mathrm{l}$ ) on the second day of implantation. The surrounding areas where the catheters were implanted showed local areas of necrotizing dermatitis, more prominent on control specimen, while areas of skin away from the exit site showed skin histology within normal limits. In control catheter samples $(C, D, E)$ there is locally extensive necrosis and heterophilic (neutrophilic) inflammation in the superficial dermis (arrow). The skin morphology adjacent to the area of necrosis and inflammation is within normal limits (arrow image E). In methylene blue catheter samples $(\mathrm{F}, \mathrm{G}, \mathrm{H})$ there is also locally extensive necrosis and heterophilic inflammation in the affected area (double arrow, image F) but to a lesser degree than in the section above and adjacent areas of skin are normal. Black arrows indicate edge of inflammation. The histological alterations in both catheter samples are consistent with acute dermal irritation and necrosis.

\section{Discussion}

The system presented is a conceptual prototype, very simple in design, which was used experimentally to show that the delivery is possible. The implementation of this technique would be more difficult in small diameter catheters than in thick catheters. Real implementation essentially would require two things: miniaturization of the reservoir and pump and addition of the external lumen to a currently existing catheter. This is mostly an engineering work and design. 
In these preliminary experiments with animal cadavers we found that the volumes that can be perfuse daily subdermally need to be necessarily small, or otherwise they will remain sub dermally forming bursa fluids. In vivo, the distribution of the fluids was sometimes circularly shaped, centered on the catheter exit site and occasionally irregular and cone-shaped with orientation towards the continuing line of the catheter that can be observed both in the dermis and the epidermis. This issue is due in part to the reduced tissue thickness of the rabbit skin and the orientation of the delivery port. Implementation in humans, where the soft tissues are thicker, and using smaller delivery ports will result in more homogeneous distribution around the interface. In both cases, however, as seen in Figures 2 and 4, the liquids reached all areas of dermis and epidermis around the catheter, guaranteeing delivery around the interface.

The histological alterations in the catheter samples were consistent with acute short-term dermal irritation. This is likely a reversible change with removal of the irritant although the inflammatory reaction is always dependent on the place of implantation and the soft tissues present [11] and is species dependent [17]. The purpose of future experiments will be then to analyze the differences in tissue response delivering, for instance, anti inflammatory.

\section{Conclusions}

It is proposed that this technology, if implemented in humans, may help prevent or reduce infections and improve the epithelial integration with percutaneous devices. The configuration presented also includes the possibility of using refillable reservoirs for chronic delivery of compounds. In the case the skin interface this possibility would be particularly useful. The technology, with proper engineered modifications, could have widespread applicability. In the area of hemo dialysis and peritoneal dialysis there is great need for improvement in implantable device - tissue interface to promote healing, maintain function and prevent or treat infection.

\section{Acknowledgments}

Funding for this project was provided by the Medical Innovation Center of the University of Michigan. We thank University of Michigan Unit of Laboratory Animal Pathology Services for help in the interpretation of the tissue sections and Professor Cynthia Marcelo for facility use. This article was made available as Open Access with the support of the University of Michigan COPE Fund, http://lib.umich. edu/cope.

\section{References}

1. Ash SR (2006) Chronic peritoneal dialysis catheters: Challenges and design solutions. Int J Artif Organs 29: 85-94.

2. Roche V (2003) Percutaneous endoscopic gastrostomy: Clinical care of PEG tubes in older adults. Geriatrics 58: 22-29.
3. Branemark R, Branemark PI, Rydevik B, Myers RR (2001) Osseo integration in skeletal reconstruction and rehabilitation: a review. J Rehabil Res Dev 38: 175-181.

4. Pendergrass CJ, Goodship AE, Blunn GW (2006) Development of a soft tissue seal around bone-anchored transcutaneous amputation prostheses. Biomaterials 27: 4183-4191.

5. Pae WE, Connell JM, Adelowo A, Boehmer JP, Korfer R, et al. (2007) Does Total Implantability Reduce Infection With the Use of a Left Ventricular Assist Device? The Lion Heart Experience in Europe. J Heart Lung Transplant 26: 219-229.

6. Thabe H, Schill S (2007) Two-Stage Reimplantation with An Application Spacer and Combined with Delivery of Antibiotics in the Management of Prosthetic Joint Infection. Oper Orthop Traumatol 19: 78-100.

7. Peramo A, Marcelo CL, Goldstein SA, Martin DC (2009) Novel organotypic cultures of human skin explants with an implant-tissue biomaterial interface. Ann Biomed Eng 37: 401-409.

8. Peramo A, Marcelo CL, Goldstein SA, Martin DC (2010) Continuous delivery of biomaterials to the skin-percutaneous device interface using a fluid pump. Artif Organs 34: E27-E33.

9. Peramo A, Marcelo CL, Goldstein SA, Martin DC (2010) Improved preservation of the tissue surrounding percutaneous devices by hyaluronic acid and dermatan sulfate in a human skin explant model. Ann Biomed Eng 38: 10981110 .

10. Peramo A, Marcelo CL (2010) Bioengineering the Skin-Implant Interface: The Use of Regenerative Therapies in Implanted Devices. Ann Biomed Eng 38: 2013-2031.

11. Rosengren A, Danielsen N, Bjursten LM (1997) Inflammatory reaction dependence on implant localization in rat soft tissue models. Biomaterials 18: 979-987.

12. Fitzpatrick N (2009) Intraosseous transcutaneous amputation prosthesis. Society of Practicing Veterinary Surgeons Review 2009.

13. Aoyama M, Mizuno T, Tatsumi E, Taenaka Y, Nemoto T et al. (2009) An Animal Study of a Newly Developed Skin-Penetrating Pad and Covering Material for Catheters to Prevent Exit-Site Infection in Continuous Ambulatory Peritoneal Dialysis. Artif Organs 33:1127-1133.

14. Cao L, Mantell S, Polla D (2001) Design and simulation of an implantable medical drug delivery system using microelectromechanical systems technology. Sensors and Actuators A (Physical) 94: 117-125.

15. Trerotola SO (2000) Hemo dialysis Catheter Placement and Management Radiology 215: 651-658.

16. Bayston R, Fisher LE, Weber K (2009) An antimicrobial modified silicone peritoneal catheter with activity against both Gram positive and Gram negative bacteria. Biomaterials 30: 3167-3173.

17. Gangjee T, Colaizzo R, von Recum AF (1985) Species-related differences in percutaneous wound healing. Annals of Biomedical Engineering 13: 451-467. 